Artículo

\title{
Efecto de un fulvato de hierro sobre calidad y producción de frutos de chile 'Serrano'
}

\author{
Rubén López-Salazar ${ }^{1}$ \\ Fidel Maximiano Peña Ramos ${ }^{1}$ \\ Francisca Sánchez-Bernal ${ }^{1}$ \\ Carlos Javier Lozano Cavazos ${ }^{2}$ \\ Adalberto Benavides Mendoza ${ }^{2}$ \\ José Antonio González-Fuentes ${ }^{2 \S}$
}

${ }^{1}$ Departamento de Horticultura-Universidad Autónoma Agraria Antonio Narro-Unidad Laguna. Periférico Raúl López Sánchez S/N, Colonia Valle Verde, Torreón, Coahuila, México. CP. 27054. (rlsmorris@hotmail.com; francis_sanchezb@hotmail.com; fperamos@gmail.com). ${ }^{2}$ Departamento de Horticultura-Universidad Autónoma Agraria Antonio Narro. Calzada Antonio Narro núm. 1923, Buenavista, Saltillo, Coahuila, México. CP. 25315. Tel. 844 4110303. (carloslozcav@gmail.com; abenmen@gmail.com).

${ }^{\S}$ Autor para correspondencia: jagf252001@gmail.com.

\section{Resumen}

Los chiles verdes en fresco, en México, son de importancia no solo porque son parte de la dieta alimenticia, sino por la mano de obra que genera su producción; sin embargo, los suelos de los estados productores presentan $\mathrm{pH}$ alcalino y esto provoca la fijación del hierro $(\mathrm{Fe})$, el cual es un elemento vital en la calidad de los frutos. Por ello, el objetivo del estudio fue determinar el efecto de un fulvato de fierro (FFe), en la calidad de frutos de chile 'serrano' para el cual se utilizaron dosis crecientes de una mezcla de ácidos fúlvicos y sulfato ferroso (FFe) a 200, 400, 600, 800, 1 000 y $1200 \mathrm{mg} \mathrm{kg}^{-1}$ adicionados en cuatro ocasiones al suelo: al momento del trasplante, a los 15, 45 y 60 ddt. y una solución nutritiva (SN) como control. Las variables de rendimiento y calidad medidas al fruto: peso fresco (PFF), peso seco (PSF), longitud (LF), diámetro (DF), firmeza (FI), sólidos solubles totales (SST) y el fierro $(\mathrm{Fe})$ y el calcio $(\mathrm{Ca})$, al tejido vegetal de follaje. Con la dosis alta de $1200 \mathrm{mg} \mathrm{kg}^{-1}$ se obtuvo el valor más alto de PFF, LF y DF, mientras que con la dosis de $1000 \mathrm{mg} \mathrm{kg}^{-1}$ se obtuvo el valor más alto de PSF, FI obtuvo el valor más alto con la dosis de $400 \mathrm{mg} \mathrm{kg}^{-1}$ y con la dosis más baja del complejo orgánico-mineral se obtuvieron los valores más altos de SST y NF. Con respecto a la concentración de nutrientes en tejido foliar la dosis de 1000 $\mathrm{mg} \mathrm{kg}^{-1}$ provoco valores óptimos de Fe y cercano al óptimo de $\mathrm{Ca}$, mientras con las dosis más bajas de 200 y $400 \mathrm{mg} \mathrm{kg}^{-1}$ la concentración de ambos minerales se presentó en exceso.

Palabras clave: Capsicum annum, chile serrano, fulvato de hierro, substancias húmicas.

Recibido: mayo de 2019

Aceptado: septiembre de 2019 


\section{Introducción}

El chile 'Serrano', es empleado en fresco y en seco debido a que contiene una cantidad de nutrientes y antioxidantes que proveen protección contra radicales libres por lo que se asocian con propiedades promotoras de la salud (Velioglu et al., 1998). Los chiles son considerados como una excelente fuente de nutrientes, así como de ácido ascórbico (vitamina C), carotenoides y compuestos fenólicos que son sus principales constituyentes antioxidantes, cuyos niveles dependen de varios factores que incluyen el cultivar, tipo de producción (orgánica o convencional), prácticas culturales, madurez del fruto y condiciones de almacenamiento (Lee y Kader 2000).

De acuerdo a Mafla y Pérez (2016), solo los frutos del género Capsicum contiene capsicina (8metil-N-vanillil-6-nonenamicida) y es empleada en la industria farmacéutica en los medicamentos que son usados contra la artritis y como antiinflamatorio. Además, los chiles son considerados dentro de la alimentación básica del pueblo mexicano y su cultivo es una fuente de empleo importante ya que requiere hasta 150 jornales por hectárea (Martínez Magdaleno et al., 1998; Inifap, 2006).

México, ocupa el tercer lugar a nivel mundial en producción de chiles verdes, después de China y Turquía ya que ellos producen 50.4 y $7.1 \%$, respectivamente (FAO-STAT, 2017). Los suelos de los estados Mexicanos productores de chile 'Serrano' como Sinaloa, Tamaulipas, San Luis Potosí y Jalisco principalmente son Calcisoles (SIAP, 2015; World Reference Base, WRB-FAOUNESCO, 2015), los que se caracterizan por poseer $\mathrm{pH}$ alcalino de 7.5 a 8, contenidos de materia orgánica que oscila alrededor del uno por ciento, más de $2 \mathrm{dS} \mathrm{m}^{-1}$ de conductividad eléctrica (CE), entre el 17 y 22 por ciento de carbonato de calcio, textura limo-arcillosa o franca y la fase de intercambio es dominada por las arcillas Illitas, lo anterior provoca que el hierro (Fe) sea fijado en este tipo de suelos y las plantas a causa de ello, entre otros factores, presentan deficiencias de este nutrimento estas, pueden ser corregidas con sales inorgánicas (fertilizantes), quelatos sintéticos y adición de materia orgánica, tanto sólida como liquida, enriquecida con este elemento (Lucena, 2004).

Las funciones del Fe en la planta, de acuerdo con Marschner (1995) son: síntesis de proteínas, como la peroxidasa para la formación de lignina y suberina; formación de la molécula de clorofila junto con el calcio, inhibe la formación del etileno e interviene en la fotorespiración y dependiendo de las características del suelo, sobre todo el $\mathrm{pH}$, las plantas desarrollan mecanismos fisiológicos para la absorción de este elemento, donde intervienen ácidos orgánicos generados por la raíz (fitosideróforos). Una forma de proporcionar $\mathrm{Fe}$ a las plantas es a través, de la adición de quelatos de este elemento; sin embargo, estos compuestos son sintéticos, difícil de conseguir y no económicos (Salisbury y Ross, 1995).

En los últimos años, con el auge de la agricultura sustentable, el uso de compuestos orgánicos como agentes complejantes, va en aumento, así el empleo de las substancias húmicas (SH) que se componen de ácidos húmicos $(\mathrm{AH})$, ácidos fulvicos $(\mathrm{AF})$ y huminas residuales (HR) son los constituyentes más significantes de la materia organica del suelo que se definen como macromoléculas orgánicas con una estructura química compleja y estable (Schnitzer, 2000; Sutton y Sposito, 2005) está muy generalizado y en una gran cantidad de países alrededor del mundo (Schnitzer, 2000). 
Los investigadores de las SH han dedicado una gran cantidad de esfuerzos, para demostrar el efecto positivo de estas en las plantas y han encontrado que la mezcla de las mencionadas substancias con nutrimentos, provocan efectos positivos en los cultivos desde el punto de vista de la nutrición y la fisiología vegetal ya que tienen un rol relevante en el ciclo de elementos minerales en el suelo (Senesi et al., 1996) y se ha reportado el incremento en longitud de tallo, raíz, hojas, masa fresca y seca y tamaño y calidad de frutos, así como rendimientos en las cosechas (Veobides-Amador et al., 2018).

Dentro de la materia orgánica del suelo, los AF son una importante fracción (Van-Hess et al., 2005) y comparados con los AH generalmente muestran una mayor actividad química y fisicoquímica (Stevenson, 1994) por lo que son considerados como parte importante de la capacidad búfer del suelo y de la retención, liberación, disponibilidad biológica y movilidad de iones metálicos en el suelo (Senesi y Miano, 1995).

Por ejemplo, Abros'kin et al. (2016), compararon la adición del complejo ácido húmico con Fe (AH-Fe) con el complejo Fe-EDTA, midieron los contenidos de Fe adicionados al suelo y por vía foliar, en raíz y tallo de las plantas de trigo y establecen que, este último complejo permanece más tiempo en la solución del suelo y los AH de complejo con Fe en la superficie de la raíz.

Asimismo, se ha demostrado el efecto sinérgico de la aplicación de sustancias fúlvicas con quelato de Fe mejorando la nutrición de plantas (Cerdan et al., 2007). Los ácidos fúlvicos en presencia de hierro producen un incremento de clorofila a y b con dosis de $5 \mu \mathrm{g} \mathrm{mL}^{-1}$ en tomate. El Fe es responsable de la síntesis de clorofila, Chen et al. (2001); Pinton et al. (1999) sugieren que el incrementos de clorofila total en hoja son evidentes con deficiencia de hierro ya que las sustancias fúlvicas mejoran la solubilidad del Fe en el medio aumentando su asimilación por las plantas.

Por lo comentado, para la producción de cultivos y obtener frutos de calidad, se hace necesaria la búsqueda de técnicas económica y ecológicamente factibles para aportar de forma simple los nutrimentos a la raíz de la planta y que ésta no invierta demasiada energía en absorber a los nutrimentos, sobre todo en suelos con $\mathrm{pH}$ alcalino, pero de manera ordenada y en las proporciones adecuadas para cada cultivo. Los AF poseen mayor acidez total, mayor cantidad de grupos funcionales carboxilos y mayor capacidad de quelatar e intercambiar cationes que los $\mathrm{AH}$ (Bocanegra et al., 2006) y llevarlos a la pared celular de la raíz para ser absorbidos por la raíz para ser transportados por la xilema hacia los puntos de demanda de las plantas (López-Salazar et al., 2014) por ello, el objetivo de este trabajo, fue determinar el efecto de un fulvato de hierro ( $\mathrm{FFe})$, en la calidad de frutos de chile 'Serrano'.

\section{Materiales y métodos}

\section{Área de estudio}

La presente investigación fue realizada en condiciones de invernadero de tecnología media, ya que éste cuenta con cortinas móviles, malla antiáfidos, extractores y calefactor, ubicado en la Universidad Autónoma Agraria Antonio Narro, en Saltillo, Coahuila, México $\left(25^{\circ} 21^{\prime}\right.$ de latitud norte y $101^{\circ} 02^{\prime}$ de longitud oeste, con la altitud de $1742 \mathrm{msnm}$ ). Durante el experimento, las temperaturas fluctuaron en el día de 21 a $35^{\circ} \mathrm{C}$ y en la noche entre 15 y $18{ }^{\circ} \mathrm{C}$. 


\section{Obtención del fulvato de hierro}

Se empleó, leonardita (mineral fósil del carbón), proporcionada por la empresa GrowMate International LLC de Houston, Texas, USA, se molió y tamizó a una malla de un milímetro de diámetro, se secó en estufa (Lab Oven, Quincy Laboratory Inc. modelo 30GC, serie G3-5572) a 70 ${ }^{\circ} \mathrm{C}$ durante $24 \mathrm{~h}$ y se dejó enfriar durante una hora, en un secador de vidrio. Posterior a esto, a $5 \mathrm{~g}$ del mineral fósil, se le adicionaron $100 \mathrm{ml}$ de hidróxido de potasio $1 \mathrm{~N}(\mathrm{KOH}, 1 \mathrm{~N})$ (CTR Scientific, Monterrey, Nuevo León, México) y se colocó a 'Baño María' (Water Bath, marca Yamato, Modelo BM 100, Japan) a $60{ }^{\circ} \mathrm{C}$ durante 120 min (López et al., 2014).

La solución se dejó enfriar durante 60 min a temperatura ambiente del laboratorio $\left(25^{\circ} \mathrm{C}\right)$, se decantó y con ácido cítrico anhidro, $1 \mathrm{~N}$ grado reactivo al $99.7 \%$ de pureza (control técnico y representaciones-CTR Scientific, Monterrey, Nuevo León, México) (López et al., 2014), se llevó la solución a pH 4 para separar los ácidos húmicos (AH) de los fúlvicos (AF) (López et al., 2014).

Los primeros fueron desechados y a estos últimos compuestos orgánicos, se les adiciono hierro al dos por ciento y como fuente de este elemento, se empleó el sulfato ferroso $\left(\mathrm{Fe}_{2} \mathrm{SO}_{4}\right)$. De esta manera, se elaboró el fulvato de Fe (FFe) además, a ambas substancias húmicas se les midió la acidez total (Schnitzer y Gupta, 1965) y el grado de humificación, mediante la Relación $\mathrm{E}_{4} / \mathrm{E}_{6}$ (Kononova, 1981) (espectrofotómetro thermo spectronic, Genesys 20, modelo 4001/4, serie LR45227, USA).

\section{Manejo del cultivo}

Semillas de chile 'Serrano', variedad 'Tampiqueño 74' (Agro Delta, Saltillo, Coahuila), fueron sembradas en charolas de poliestireno de 200 cavidades, con el sustrato 'peat moss' (Premier Horticulture Inc. Quakertown, PA USA) y 'perlita' (Hortiperl, Termolita SAPI de CV Santa Catarina, Nuevo León, México) (relación 1:1 v/v); a los 35 días después de siembra (dds), cuando las plántulas tuvieron tres pares de hojas verdaderas y 15 a $20 \mathrm{~cm}$ de longitud, fueron trasplantadas en macetas de plástico que contenían $25 \mathrm{~kg}$ del horizonte Ap de un Calcisol obtenido del estado de Coahuila. Las características principales del suelo empleado se presentan en el Cuadro 1.

Cuadro 1. Características físicas y químicas del horizonte Ap del suelo Calcisol empleado en el experimento. $\mathrm{MO}=$ materia orgánica; $\mathrm{CO}_{3}=$ carbonatos; $\mathrm{CE}=$ conductividad eléctrica; CIC = capacidad de intercambio catiónico.

\begin{tabular}{lcccccccc}
\hline Característica & $\begin{array}{c}\text { Arena } \\
(\%)\end{array}$ & $\begin{array}{c}\text { Arcilla } \\
(\%)\end{array}$ & $\begin{array}{c}\text { Limo } \\
(\%)\end{array}$ & $\begin{array}{c}\mathrm{MO} \\
(\%)\end{array}$ & $\begin{array}{c}\mathrm{pH} \\
(1: 2 \text { agua })\end{array}$ & $\begin{array}{c}\mathrm{CO}_{3} \\
(\%)\end{array}$ & $\mathrm{CE}\left(\mathrm{dS} \mathrm{m}^{-1}\right)$ & $\mathrm{CIC}\left(\mathrm{cmol}_{\mathrm{c}} \mathrm{kg}^{-1}\right)$ \\
Horizonte Ap & 37.48 & 22.52 & 40 & 3.41 & 8.57 & 52.7 & 1.1 & 78.1 \\
\hline
\end{tabular}

Las plantas fueron distribuidas a una distancia de $60 \mathrm{~cm}$ entre ellas y a $80 \mathrm{~cm}$ entre hileras. A los tres días después del trasplante (ddt), las plantas en macetas fueron fertilizadas con la solución nutritiva (SN) Steiner (Steiner, 1961) la cual se preparó en base a un análisis químico del agua de riego (Cuadro 2) y usando fertilizantes solubles grado técnico como ácido fosforico $\left(\mathrm{H}_{3} \mathrm{PO}_{4}\right)$, ácido bórico $\left(\mathrm{H}_{3} \mathrm{BO}_{3}\right)$, sulfato ferroso $\left(\mathrm{FeSO}_{4}\right)$, sulfato de cobre $\left(\mathrm{CuSO}_{4}\right)$ y sulfato de zinc $\left(\mathrm{ZnSO}_{4}\right)(\mathrm{CTR}$ Scientific, Monterrey, Nuevo León, México), nitrato de potasio $\left(\mathrm{KNO}_{3}\right)$, nitrato de calcio $\left(\mathrm{Ca}\left(\mathrm{NO}_{3}\right)_{2}\right)$, nitrato de magnesio $\left(\mathrm{Mg}\left(\mathrm{NO}_{3}\right)_{2}\right)$ (Keswick SA de CV, Saltillo, Coahuila, México). 
Cuadro 2. Características del agua de riego (A 1) y de las soluciones nutritivas, solución 1 (SN 1) y solución 2 (SN 2) adicionadas al chile 'Serrano', variedad 'Tampiqueño 74'.

\begin{tabular}{ccccccccccccccc}
\hline & $\mathrm{pH}$ & $\begin{array}{c}\left.\mathrm{CE}_{(\mathrm{dS} \mathrm{m}}^{-1}\right) \\
\mathrm{NO}_{3}\end{array}$ & $\mathrm{H}_{2} \mathrm{PO}_{4}$ & $\mathrm{Cl}$ & $\mathrm{SO}_{4}$ & $\mathrm{HCO}_{3}$ & $\mathrm{~K}$ & $\mathrm{Ca}$ & $\mathrm{Mg}$ & $\mathrm{Fe}$ & $\mathrm{Cu}$ & $\mathrm{Zn}$ & $\mathrm{B}$ \\
\hline $\mathrm{A} \mathrm{1}$ & 7.1 & & & & 0.7 & 0.16 & 0.78 & 0.28 & 1.14 & 0.2 & & & & \\
$\mathrm{SN} 1$ & 5.7 & 1.3 & 7.2 & 1.3 & & 4.4 & 0.7 & 4.3 & 4.7 & 2.4 & 2 & 1.9 & 2 & 0.3 \\
$\mathrm{SN} 2$ & 5.5 & 2.6 & 14.3 & 2.6 & & 8.9 & 0.7 & 8.8 & 10.6 & 5 & 2.5 & 2.2 & 2.5 & 0.5 \\
\hline
\end{tabular}

Las unidades de los aniones: nitratos $\left(\mathrm{NO}_{3}\right)$; fosfatos $\left(\mathrm{H}_{2} \mathrm{PO}_{4}\right)$; cloruros $(\mathrm{Cl})$; sulfatos $\left(\mathrm{SO}_{4}\right)$; bicarbonatos $\left(\mathrm{HCO}_{3}\right)$ y la de los cationes: potasio $(\mathrm{K})$, calcio $(\mathrm{Ca})$ y magnesio $(\mathrm{Mg})$ son meq $\mathrm{L}^{-1}$. Y las de fierro $(\mathrm{Fe})$, cobre $(\mathrm{Cu})$, zinc $(\mathrm{Zn})$ y boro (B), son en $\mathrm{mg} \mathrm{L}^{-1}$.

Los tratamientos del FFe adicionados al suelo fueron: 200, 400, 600, 800, 1000 y $1200 \mathrm{mg} \mathrm{kg}^{-1}$ por litro de solución nutritiva con cinco repeticiones y como control se aplicó solamente la SN Steiner completa con una conductividad eléctrica de $2 \mathrm{dS} \mathrm{m} \mathrm{m}^{-1}$. La cantidad de solución nutritiva que se adiciono en cada riego fue de acuerdo a la cantidad perdida por evapotranspiración utilizando la diferencia de peso en $24 \mathrm{~h}$ la cual se midió cada semana para hacer los ajustes correspondientes debido al incremento de la planta y en consecuencia al aumento de evapotranspiración.

Para evitar acumulación de sales en el medio de crecimiento se adicionó en cada riego una fracción de lavado que origino en cada riego un drenaje de $25 \%$. Los tratamientos fueron adicionados en cuatro ocasiones: al momento del trasplante, a los 15, 45 y 60 ddt. El manejo de fitosanitario del cultivo fue mediante un calendario de aplicaciones preventivas quincenales utilizando productos amigables al medio ambiente como extracto de ajo, aceite de neem y sulfato de cobre entahidratado.

Métodos utilizados para las determinaciones: MO por Walkey-Black; $\mathrm{pH}$ por el método potencio métrico, CE mediante electrodo de conductimetro, CIC método de acetato amónico $1 \mathrm{~N}, \mathrm{pH} 7 \mathrm{y}$ $\mathrm{CO}_{3}$ método de Bernard por desprendimiento de $\mathrm{CO}_{2}$ en calcímetro, porcentajes de arena limo y arecilla por medio de la velocidad de sedimentación de las partículas.

En el Cuadro 3, se aprecia la alta acidez total (AT) de los ácidos fúlvicos (AF); es decir, mayor cantidad de grupos funcionales oxigenados, con estructura poco condensada y poseen mayor cantidad de cadenas alifáticas.

Cuadro 3. Acidez total (AT), grupos funcionales carboxilo (-COOH), oxhidrilos y relación de absorbancias a 465 y $665 \mathrm{~nm}\left(\mathbf{E}_{4} / \mathbf{E}_{6}\right)$ de ácidos fúlvicos obtenidos de leonardita.

\begin{tabular}{ccccc}
\hline Substancia húmica & $\mathrm{AT}\left(\mathrm{cmol}_{\mathrm{c}} \mathrm{kg}^{-1}\right)$ & $-\mathrm{COOH}^{-}\left(\mathrm{cmol}_{\mathrm{c}} \mathrm{kg}^{-1}\right)$ & $-\mathrm{OH}^{-}\left(\mathrm{cmol}_{\mathrm{c}} \mathrm{kg}^{-1}\right)$ & Relación $\mathrm{E}_{4} / \mathrm{E}_{6}$ \\
\hline $\mathrm{AF}$ & 1277 & 561 & 716 & 5.7 \\
\hline
\end{tabular}

$\mathrm{AF}=$ ácidos fúlvicos.

\section{Variables de calidad cuantificadas}

Los frutos de chiles fueron cosechados a madurez comercial, se realizaron tres cortes espaciados por 25 días cada uno, siendo el primer corte a los $117 \mathrm{ddt}$. Las variables medidas de rendimiento y calidad fueron: peso fresco (PF), longitud (LF), diámetro ecuatorial (DE) (Vernier Stainless-Steel, 
Marca Truper) y firmeza (FF) (penetrometro, fruit hardess tester, modelo FHT 200. Extech, instruments), sólidos solubles totales (SST) (Master Refractometer, Marca Atago), número de frutos (NF), peso seco (PSF) y en el tejido vegetal de follaje, los contenidos de Fe y Ca (digestión por vía húmeda-digestor a base de microondas, marca Cem, Modelo MARS 6 One Touch Technology, USA y la medición con un espectofotómetro de absorsión atómica, marca Varian, modelo A5) (Lorenzo et al., 2010).

El experimento fue establecido en un diseño experimental completamente al azar, con siete tratamientos y cinco repeticiones. Los resultados obtenidos fueron analizados mediante un análisis de varianza (Anva) y para separación de medias se utilizó la prueba de Tukey $(p \leq 0.05)$, utilizando el paquete estadístico Minitab, versión 17 en español para Windows.

\section{Resultados y discusión}

Para peso del fruto (PF), no se encontraron diferencias estadísticas significativas; sin embargo, numéricamente (Cuadro 4), se aprecia que al adicionar las dosis de 200 a $800 \mathrm{mg} \mathrm{kg}^{-1} \mathrm{del} \mathrm{FFe}$ conforme aumentó la cantidad los valores disminuyeron; pero, al adicionar $1200 \mathrm{mg} \mathrm{kg}^{-1} \mathrm{del} \mathrm{FFe}$ se presentó numéricamente el mayo PF, ya que sobrepasó $84 \%$ a las plantas testigo las cuales se cultivaron solo con solución nutritiva (SN). Esto fue debido posiblemente a que las plantas con que recibieron este tratamiento fueron frutos más grandes con mayor diámetro y longitud, pero en cantidad fueron menos como se aprecia en el Cuadro 4, lo que sugiere que la haber menos cantidad alcanzaron mayor tamaño y peso de fruto.

Cuadro 4. Calidad de frutos de chile serrano, variedad 'Tampiqueño 74' con la adición de un fulvato de fierro a diferentes concentraciones.

\begin{tabular}{|c|c|c|c|c|c|c|c|}
\hline Tratamiento & $\mathrm{PF}(\mathrm{g})$ & PSF (g) & $\mathrm{LF}(\mathrm{cm})$ & $\mathrm{DF}(\mathrm{cm})$ & $\mathrm{FI}(\mathrm{N})$ & SST ( ${ }^{\circ}$ Brix) & $\mathrm{NF}\left(\mathrm{N}^{\circ}\right)$ \\
\hline $\mathrm{FFe} 2$ & $380.3^{\mathrm{a}}$ & $18.17^{\mathrm{ab}}$ & $9.32^{\mathrm{a}}$ & $1.2^{\mathrm{a}}$ & $11.88^{c}$ & $6.85^{\mathrm{a}}$ & $35.58^{\mathrm{a}}$ \\
\hline $\mathrm{FFe} 4$ & $430.4^{\mathrm{a}}$ & $19^{\mathrm{ab}}$ & $11.26^{\mathrm{a}}$ & $1.47^{\mathrm{a}}$ & $17.33^{\mathrm{a}}$ & $6.37^{\mathrm{a}}$ & $23.74^{\mathrm{ab}}$ \\
\hline FFe6 & $376.9^{a}$ & $14.7^{\mathrm{ab}}$ & $9.86^{\mathrm{a}}$ & $1.3^{\mathrm{a}}$ & $13.43^{b c}$ & $6.7^{\mathrm{a}}$ & $31.66^{\mathrm{ab}}$ \\
\hline FFe8 & $342.9^{\mathrm{a}}$ & $15.92^{\mathrm{ab}}$ & $9.48^{\mathrm{a}}$ & $1.47^{\mathrm{a}}$ & $13.9^{\mathrm{bc}}$ & $6^{\mathrm{a}}$ & $22.25^{\mathrm{ab}}$ \\
\hline $\mathrm{FFe} 10$ & $421.3^{\mathrm{a}}$ & $23.66^{\mathrm{a}}$ & $10.79^{a}$ & $1.46^{\mathrm{a}}$ & $13.57^{\mathrm{bc}}$ & $6^{\mathrm{a}}$ & $17.55^{\mathrm{b}}$ \\
\hline $\mathrm{FFe} 12$ & $452.3^{\mathrm{a}}$ & $14.32^{\mathrm{b}}$ & $11.53^{\mathrm{a}}$ & $1.6^{\mathrm{a}}$ & $13.2^{\mathrm{bc}}$ & $6.18^{\mathrm{a}}$ & $21.95^{\mathrm{ab}}$ \\
\hline SN & $245.2^{\mathrm{a}}$ & $13.67^{\mathrm{b}}$ & $9.67^{\mathrm{a}}$ & $1.37^{\mathrm{a}}$ & $14.98^{\mathrm{ab}}$ & $6.39^{\mathrm{a}}$ & $25.16^{\mathrm{ab}}$ \\
\hline
\end{tabular}

$\mathrm{PF}=$ peso fresco fruto; $\mathrm{PSF}=$ peso seco fruto; $\mathrm{LF}=$ longitud fruto; $\mathrm{DF}=$ diámetro fruto; $\mathrm{FI}=$ firmeza fruto; $\mathrm{SST}=$ sólidos solubles totales; $\mathrm{NF}=$ número de frutos y $\mathrm{SN}=$ solución nutritiva. Valores medios de cada tratamiento con superíndices de letras iguales dentro de cada columna no son estadísticamente diferentes (Tukey $p \leq 0.05$ ).

Los tratamientos, no provocaron efecto significativo en la longitud del fruto (LF); sin embargo, para esta variable, desde la dosis más baja hasta la penúltima, los valores oscilaron por 2.2 unidades; es decir, muy poca variación; sin embargo, al aplicar la dosis mayor del FFe se superó numéricamente al control 23\%. Para el diámetro del fruto (DF) el comportamiento fue de forma similar que en la variable anterior; es decir, los tratamientos no provocaron efecto significativo; sin embargo, la dosis del FFe a $1200 \mathrm{mg} \mathrm{kg}^{-1}$, superó a la SN en $33 \%$. 
La firmeza (FI) medida en Newtons, es una variable de calidad muy importante, ya que en gran medida la vida de anaquel depende de ella; en esta variable de respuesta a la adición de los tratamientos y sobresale la adición de $400 \mathrm{mg} \mathrm{kg}^{-1}$ del FFe, ya que aventajó al control 16\% y a la dosis de $200 \mathrm{mg} \mathrm{kg}^{-1}$ la superó $45 \%$ siendo esta diferencia altamente significativa.

Para sólidos solubles totales (SST), no se encontró diferencia significativa a la aplicación de los tratamientos; sin embargo, numéricamente el valor mayor en esta variable se obtuvo con la adición de $200 \mathrm{mg} \mathrm{kg}^{-1}$ el cual superó al testigo con $7 \%$ más. En general los chiles serranos comercialmente se cosechan con valores de entre 5 a $6{ }^{\circ}$ Brix (Gómez y Gómez et al., 2017) y los valores que se obtuvieron en el presente estudio oscilaron entre 6 y 6.8 .

En el Cuadro 4, se observó que en el número de frutos (NF) los tratamientos provocaron efecto significativo, con la adición de la dosis inferior del FFe de $200 \mathrm{mg} \mathrm{kg}^{-1}$ se obtuvo el valor más alto, siendo superior en $41 \%$ comparado con a la SN. Asimismo, la dosis más baja de $200 \mathrm{mg} \mathrm{kg}^{-1} \mathrm{supero}$ significativamente en $102 \%$ a la dosis de $1000 \mathrm{mg} \mathrm{L}^{-1}$. Los valores con los otros tratamientos no fueron diferentes entre sí. Para la variable de peso seco del fruto (PSF), los tratamientos provocaron un efecto significativo, al adicionar el tratamiento con la dosis de $1000 \mathrm{mg} \mathrm{kg}^{-1}$, se superó al testigo (SN) en $73 \%$. El resto de los tratamientos, aunque no fueron significativamente diferentes, numéricamente todos fueron superiores al testigo y es importante mencionar que las dosis bajas de 200 y $400 \mathrm{mg} \mathrm{kg}^{-1}$ superaron al testigo 33 y $38 \%$ respectivamente.

En el Cuadro 5, se presenta el contenido de hierro $(\mathrm{Fe})$ y calcio $(\mathrm{Ca})$ del tejido vegetal, en donde los tratamientos afectaron significativamente a estos nutrimentos. Con la dosis más baja del FFe, se presentaron los valores superiores en ambos elementos y conforme se aumentó la dosis, los valores disminuyeron considerablemente. Las plantas donde se adicionaron 200 y $400 \mathrm{mg} \mathrm{kg}^{-1} \mathrm{del}$ FFe no fueron deficientes en Fe; sin embargo, con el resto de los tratamientos si lo fueron (Reuter y Robinson, 1997), señalan que las cantidades adecuadas de este elemento en el tejido vegetal de follaje, oscila entre 60 y $80 \mathrm{mg} \mathrm{kg}^{-1}$.

Cuadro 5. Análisis nutrimental de Fe y Ca del tejido vegetal de follaje de chile 'Serrano', variedad 'Tampiqueño 74'.

\begin{tabular}{cccccccc}
\hline Tratamiento & FFe2 & FFe4 & FFe6 & FFe8 & FFe10 & FFe12 & SN \\
\hline $\mathrm{Fe}^{2+}\left(\mathrm{mg} \mathrm{kg}^{-1}\right)$ & $145^{\mathrm{a}}$ & $137.5^{\mathrm{a}}$ & $45^{\mathrm{b}}$ & $42.5^{\mathrm{b}}$ & $60^{\mathrm{b}}$ & $45^{\mathrm{b}}$ & $50^{\mathrm{b}}$ \\
$\mathrm{Ca}^{2+}(\%)$ & $3.34^{\mathrm{a}}$ & $3.13^{\mathrm{a}}$ & $1.53^{\mathrm{b}}$ & $0.99^{\mathrm{b}}$ & $1.27^{\mathrm{b}}$ & $0.96^{\mathrm{b}}$ & $0.97^{\mathrm{b}}$ \\
\hline
\end{tabular}

Valores medios de cada tratamiento con superíndices de letras iguales dentro de cada columna no son estadísticamente diferentes (Tukey $p \leq 0.05$ ).

El principal elemento antagonista con el Fe es el Ca y en este experimento al agregar 200, $400 \mathrm{y}$ $600 \mathrm{mg} \mathrm{kg}^{-1}$ del FFe las plantas mostraron valores dentro de los rangos suficientes sobre $1.3 \%$, de acuerdo con los mismos autores, mientras que con el resto de los tratamientos fueron deficientes.

Los resultados encontrados en este estudio en particular con la dosis alta de $1200 \mathrm{mg} \mathrm{kg}^{-1}$ contrastan con los resultados de (Adani et al., 1998), quienes reportaron que con la aplicación de sustancias húmicas y fulvicas la disponibilidad de hierro en el medio de crecimiento incrementa debido a una reducción de $\mathrm{Fe} 3+$ a Fe2 + lo que provoca un incremento de este elemento en la planta. 
Autores como Bocanegra et al. (2006), establecieron que los ácidos fúlvicos (AF) tienen alta acidez total, gran número de grupos funcionales carboxilo, alta capacidad de adsorción y de intercambiar cationes, mayor que los ácidos húmicos $(\mathrm{AH})$, porque, los $\mathrm{AF}$ son responsables de la quelatación y movilización de iones metálicos, lo que incluye al Fe y aluminio ( $\mathrm{Al}$ ) además, la capacidad de quelatar nutrimentos como el Fe y moverlo; a través, de la membrana sugiere que éstos juegan un rol como agentes quelatantes naturales en la movilización y transporte de Fe y otros micronutrientes.

Esto concuerda con los AF mezclados con el Fe, adicionados en este trabajo. Álvarez-Fernández et al. (2006), señalan que la deficiencia de Fe en cultivos, provoca una disminución en el tamaño de los frutos y su firmeza, lo que redunda en la apariencia esto es, afecta la vida de anaquel de los frutos y por consiguiente la calidad, por lo que, la forma más empleada para corregir esta deficiencia es el uso de quelatos de hierro; sin embargo, estos productos son costosos y la cantidad a adicionar al suelo y por vía foliar es alta y su efectividad, depende de diversos factores.

El precio de una libra de un quelato de hierro, oscila entre 40 y 50 dólares americanos y dependiendo del cultivo se deben adicionar de dos a tres veces durante el ciclo, mientras que el costo de un galón de un FFe varía entre 20 y 22 dólares americanos y solo se deben adicionar entre una o dos veces máximo.

Ante esta situación, el uso de las substancias húmicas $(\mathrm{SH})$ se perfilan como algo viable, ya que, como característica fundamental, es que poseen grupos funcionales oxigenados $\left(-\mathrm{COOH}^{-},-\mathrm{OH}^{-}\right.$, $\mathrm{COO}^{-}$) y nitrogenados $\left(\mathrm{NH}^{-}, \mathrm{NH}_{2}\right)$. De estos dos tipos de grupos funcionales, los primeros forman más de $80 \%$ de la molécula de las substancias orgánicas mencionadas y con capacidad de intercambiar cationes hasta $1200 \mathrm{cmolc} \mathrm{kg}^{-1}$ (Schnitzer, 2000), que tienen la particularidad de complejar y quelatar a los nutrimentos (cationes).

Dado su pequeño tamaño molecular, los AF pueden pasar a través de microporos de sistemas biológicos o membranas artificiales y los $\mathrm{AH}$ no pueden por su mayor tamaño. La capacidad combinada de los AF de quelatar nutrimentos como el Fe y moverse; a través, de las membranas, sugiere que estos compuestos pueden desempeñar un rol similar a quelatantes naturales en la movilización y transporte del Fe y otros micronutrimentos. En un estudio en girasol aplicando una solución Hoagland con isotopo ${ }^{59} \mathrm{Fe}$ los AF funcionaron como agentes quelatantes y el Fe presentó mayor disponibilidad e incremento para la planta (Bocanegra et al., 2006).

Investigaciones realizadas por Aminifard et al. (2012), aplicaron AF a chile para determinar el efecto en la calidad y encontraron que especialmente los compuestos orgánicos, aumentan la actividad antioxidante también, los sólidos solubles totales, los carbohidratos totales y la capsaicina. En tratamiento de arroz con AF en suelos calcáreos, aumenta la disponibilidad del Fe y la eficiencia de la mezcla Fe-AF, como fertilizante fue mayor que el $\mathrm{FeCl}_{3}$ (Pandeya et al., 1998); Sánchez-Sánchez et al. (2002), reportaron en árboles de limón un incremento en la disponibilidad foliar de $\mathrm{Fe}$ y $\mathrm{Cu}$ cuando aplicaron en dos ocasiones $\mathrm{SH}$ que contenían $90.7 \%$ de AF.

Por su parte Abros'kin et al. (2016), adicionaron Fe con EDTA y ácidos húmicos (AH) a semillas de trigo y encontraron que al aplicar AH con Fe, aumentó el contenido de clorofila de la hoja y el contenido de Fe fue mayor en la raíz que en el tallo y en la hoja, esto posiblemente fue debido a que el complejo Fe-AH se acumuló en la superficie de la raíz, lo que limito la absorción y posterior translocación del elemento y esto, trae como consecuencia la limitación del metabolismo de lípidos. 
En el presente trabajo, se encontró que en el tejido vegetal de follaje con la adición de las dos dosis más bajas de 200 y $400 \mathrm{mg} \mathrm{kg}^{-1}$ del FFe, se presentó la concentración de Fe en cantidades excesivas de 145 y $137.5 \mathrm{mg} \mathrm{kg}^{-1}$ respectivamente ya que, de acuerdo a Reuter y Robinson (1997) la concentración adecuada de Fe es de 60 a $80 \mathrm{mg} \mathrm{kg}^{-1}$ además, al aplicar $1000 \mathrm{mg} \mathrm{kg}^{-1}$ del FFe, la cantidad del Fe se ubicó dentro del rango óptimo con $60 \mathrm{mg} \mathrm{kg}^{-1}$. En referencia el calcio de acuerdo a Reuter y Robinson (1997) la concentración adecuada es de 1.3 a $2.5 \%$ y con esta misma dosis de $1000 \mathrm{mg} \mathrm{kg}^{-1}$ del FFe, la concentración de Ca se presentó cercana al óptimo con $1.27 \%$ y con las dosis bajas de 200 y $400 \mathrm{mg} \mathrm{kg}^{-1}$ las concentraciones fueron de 3.34 y $3.13 \%$.

Lo anterior indica que con la adición de ácidos fulvicos, a las bajas concentraciones estudiadas, los contenidos minerales aumentan lo que sugiere que podría aportar beneficios como en vida de anaquel, como sugiere Luchsinger et al. (2006) que al aumentar la concentración de calcio la firmeza de frutos aumenta.

Lo anterior, denota que el Fe incremento en follaje cuando se adicionaron las dos dosis más bajas del complejo orgánico-mineral como lo reportan Karakurt et al. (2009), así como Ertani et al. (2015). Además, Aminifard et al. (2012) reportan que con la dosis más baja de $25 \mathrm{mg} \mathrm{kg}^{-1} \mathrm{de}_{\text {ácidos }}$ fulvicos aplicadas a chiles (Capsicum annuиm L.) obtuvieron mayor calidad de fruta cosechada $\mathrm{y}$ mayor contenido de compuestos antioxidantes en frutos y con la dosis más alta de $250 \mathrm{mg} \mathrm{kg}^{-1} \mathrm{se}$ obtuvo mayor contenido de capsicina en los frutos de chile.

Esto coincide con lo reportado por Oliver (2012) quien reporta que con la adición de SH con Fe, la concentración en tejido foliar de este elemento aumenta de forma considerable por arriba del optimo cuando hay baja disponibilidad de este en el medio de crecimiento. Con esto, se pone de manifiesto el efecto de las bajas concentraciones aplicadas del FFe al evaluar las variables de calidad medidas al fruto de chile 'Serrano', con excepción del peso fresco, peso seco y la longitud del fruto, ya que, en estas variables medidas, quien provoco un efecto significativo fue la dosis superior del compuesto orgánico-mineral.

\section{Conclusiones}

El Fulvato de hierro (FFe), provoco un efecto positivo en las variables de calidad medidas al chile 'Serrano', cultivado en un suelo calcisol, ya que con la adición de la dosis FFe10 se obtuvo el mayor peso seco de frutos, con el menor número de frutos por planta y el valor más bajo en grados Brix, en contraste con la dosis más baja FFe2 se obtuvo el mayor número de frutos con el menor tamaño, pero el mayor valor en grados Brix. Para el contenido mineral en tejido vegetal, con las dos dosis inferiores FFe2 y FFe4 se encontraron los valores más altos de Fe y Ca.

El uso de sustancias fulvicas en combinación con productos inorgánicos se obtienen de un procedimiento sencillo y a bajo costo ya que las materias primas como leonardita son abundantes, baratas y susceptibles a modificación química por lo que es una forma prometedora de mejorar la calidad de las cosechas de chile serrano por medio del estímulo de crecimiento aumentando la eficiencia de algunos nutrientes minerales. 


\section{Literatura citada}

Abros'kin, D. P.; Fuentes, M.; Garcia, M. J. M.; Klyain, O. I.; Senik, S. V.; Volkov, D. S.; Perminova, I. V. and Kulikova, N. A. 2016. The effect of humic acids and their complexes with Iron on the functional status of plants grown under iron deficiency. Eurasian Soil Sci. 49(10):1099-1108.

Adani, F.; Genevini, P.; Zaccheo, P. and Zocchi, G. 1998. The effect of commercial humic acid on tomato plant growth and mineral nutrition. J. Plant Nutr. 21(3):561-575.

Álvarez, F. J. and Abadía, A. 2006. Iron deficiency, fruit yield and fruit quality. In: Barton, L. L. and Abadía, J. (Eds.), iron nutrition in plants and Rhizospheric microorganisms. Springer, Dordrecht, Netherlands. 85-101 pp.

Aminifard, M. H.; Aroiee, H.; Nemati, H.; Azizi, M. and Jaafar H. Z. E. 2012. Fulvic acid affects pepper antioxidant activity and fruit quality. Afr. J. Biotechnol. 11(68):13179-13185.

Bocanegra, M. P.; Lobartini, J. C. and Orioli, G. A. 2006. Plant uptake of iron chelated by humic acids of different molecular weights. Commun Soil Sci. Plant Anal. 37(1-2):239-248.

Cerdán, M.; Sánchez, S. A.; Juárez, M.; Sánchez, A. J.; Jordá, J. and Bermúdez, D. 2007. Partial replacement of $\mathrm{Fe}$ (o,o-EDDHA) by humic substances for Fe nutrition and fruit quality of citrus. J. Plant Nutr. Soil Sci. 170(4):474-478.

Chen, Y.; Magen, H. and Clapp, C. E. 2001. Plant growth stimulation by humic substances and their complexes with iron. In: Proceedings of the Dalia Greidinger Symposium. The International Fertiliser Society, Lisbon, Portugal. 1-14 pp.

Ertani, A.; Sambo, P.; Nicoletto, C.; Santagata, S.; Schiavon, M. and Nardi, S. 2015. The use of organic biostimulants in hot pepper plants to help low input sustainable agriculture. Chem. Biol. Technol. Agric. 2(11):1-10.

FAO-STAT. Food and Agriculture Organization of the United Nations (FAO). 2017. http://www.fao.org/faostat.

Gómez y Gómez, Y.; Gumecindo, C.; Bautista, E. y Torres, L. G. 2017. Elaboración de una película comestible incluyendo principios activos vegetales para la protección del chile serrano. Conferencia SNIQBA, Tlaxcala, Tlaxcala. 2 p.

INIFAP. 2006. Tecnología en producción chiles seco. Instituto nacional de investigaciones forestales, agrícolas y pecuarias. Centro de Investigación Regional Norte Centro- Campo Experimental Zacatecas. Libro técnico núm. 5. 63-64 pp.

Karakurt, Y.; Unlu, H.; Unlu, H. and Padem, H. 2009. The influence of foliar and soil fertilization of humic acid on yield and quality of pepper. Acta Agric. Scand. Sect. B: Soil Plant Sci. 59(3):233-237.

Kononova, M. M. 1981. Materia orgánica del suelo. Nueva enciclopedia de agricultura: su naturaleza, propiedades y métodos de investigación. Ed. Oikos-Tan. Madrid, España. 544 p.

Lee, S. K. and Kader, A. A. 2000. Preharvest and postharvest factors influencing vitamin C content of horticultural crops. Postharvest Biology Biotechnolgy. 20:207-220.

López, S. R.; González, C. G.; Vázquez, A. R. E.; Olivares, S. E.; Vidales, C. J. A.; Carranza de la Rosa, R. y Ortega, E. M. 2014. Metodología para obtener ácidos húmicos y fulvicos y su caracterización mediante espectrofotometría infrarroja. Rev. Mex. Cienc. Agríc. 8(Pub. Esp.):1397-1407.

Lorenzo, M.; Reyes, A.; Blanco, I. y Vasallo, M. C. 2010. Determinación de $\mathrm{Ca}, \mathrm{Cu}, \mathrm{Fe}$ y $\mathrm{Pb}$ por espectrofotometría de absorción atómica en aguardientes de caña. ICIDCA. 44(3):3-6. 
Lucena, J. J. 2004. Quelatos de hierro conteniendo o, pEDDHA. Estudios sobre su eficacia. Phytoma. 163:26-32.

Luchsinger, L.; Soto, M. y Báez, S. R. 2006. Efecto de aspersiones de calcio en precosecha sobre la calidad postcosecha en frutos de frambuesa "Chilliwack". Rev. Iberoam. Tecnol. Postcos. Hermosillo, México. 7(2):142-149.

Mafla, H. L. F. y Pérez, N. M. G. 2016. Utilización de Jalapeño (Capsicum annuum), pimiento verde (Capsicum annuun L.) y espinaca (Spinacia eleracea L.) como fuentes de antioxidantes en la elaboración de una salsa picante. Tesis (Ingeniera en alimentos), Universidad San Francisco de Quito, Colegio de Ciencias e Ingeniería; Quito, Ecuador. 1215 pp. 2016. http://repositorio.usfq.edu.ec/handle/23000/5166.

Marschner, H. 1995. Functions of mineral nutrients: micronutrients. Iron, In: mineral nutrition of higher plants, Academic Press, Cambridge, UK. 313-324 pp.

Martínez, M. R. A.; Narro, R. J. G.; Martínez, R. D. y Orejón, G. E. 1998. Producción y comercialización de Chile Serrano (Capsicum annum L.) en el ejido la leona, municipio de Ramos Arizpe. Tesis de Licenciatura. Universidad Autónoma Agraria Antonio Narro (UAAAN). Saltillo, Coahuila, México. 20-22 pp.

Oliver, A. M. 2012. Efectos de las sustancias húmicas sobre los mecanismos de toma de hierro en plántulas de tomate. Tesis Doctoral, Universidad de Alicante.

Pandeya, S. B.; Singh, A. K. and Dhar, P. 1998. Influence of fulvic acid on transport of iron in soils and uptake by paddy seedlings. Plant Soil. 198:117-125.

Pinton, R.; Cesco, S.; Santi, S.; Agnolon, F. and Varanini, Z. 1999. Water-extractable humic substances enhance iron deficiency responses by Fe-deficient cucumber plants. Plant Soil. 210:145-157.

Reuter, D. J.; Robinson, J. B. and Dutkiewicz, C. 1997. Plant analysis ð an interpretation manual. CSIRO, Melbourne, Australia. 572 pp.

Salisbury, F. B. and Ross, C. W. 1995. Fisiologi Tumbuhan Jilid 1. Bandung: ITB.

Sánchez-Sánchez, A.; Sánchez-Andreu, J.; Juárez, M.; Jorda, J. and Bermúdez, D. 2002. Humic substances and aminoacids improve effectiveness of chelate FeEDDHA in lemon trees. J. Plant Nutr. 25:2433-2442.

Schnitzer, M. 2000. A lifetime perspective on the chemistry of soil organic matter. Adv. Agron. 68:1-58.

Schnitzer, M. and Gupta, U. C. 1965. Determination of acidity in soil organic matter. Soil Sci. Soc. Am. Proc. 29:274-277.

Senesi, N. and Miano, T. M. 1995. The rol of abiotic interactions with humic substances on the environmental impact of organic pollutants. Natural Antrhropogenic Organics. 1:311-335.

Senesi, N.; Miano, T. M. and Brunetti, G. 1996. Humic-like substances in organic amendments and effects on native soil humic substances. In: Piccolo, A. (Ed.). Humic Substances in Terrestrial Ecosystems. USA, New York. Elsevier.

SIAP. 2015. Márgenes de comercialización de chile Serrano. Servicio de información agroalimentaria y pesquera.

Steiner, A. A. 1961. A universal method for preparing nutrient solutions of a certain desired composition. Plant Soil. 15:134-154.

Stevenson, F. J. 1994. Humus chemistry: genesis, composition, reactions. USA, New York Jhon Wiley and Sons, Interscience Press.

Sutton, R. and Sposito, G. 2005. Molecular structure in soil humic substances: the new view. Environ. Sci. Technol. 23:9009-9015. 
Van-Hees, P. A.; Jones, D. L.; Finaly, R.; Godbold, D. L. and Lundstrom, U. S. 2005. The carbon we do not see the impact of low molecular weight compounds on carbon dynamics and respiration in forest soils: a review. Soil Biol. Biochem. 37:1-13.

Velioglu, Y. S.; Mazza, G. and Oomah, B. D. 1998. Antioxidant activity and total phenolics in selected fruits, vegetables, and grain products. J. Agric. Food Chem. 46:4113-4117.

Veobides-Amador, H.; Gurdi-Izquierdo, F. and Vázquez-Padrón, V. 2018. Las sustancias húmicas como bioestimulantes de las plantas bajo condiciones de estrés ambiental. Revisión bibliográfica. Cultivos tropicales. 39:102-109.

WRB. 2015. World Reference Base for Soil Resources. Food and Agriculture Organization of United Nations (FAO/UNESCO). Rome, Italy. 